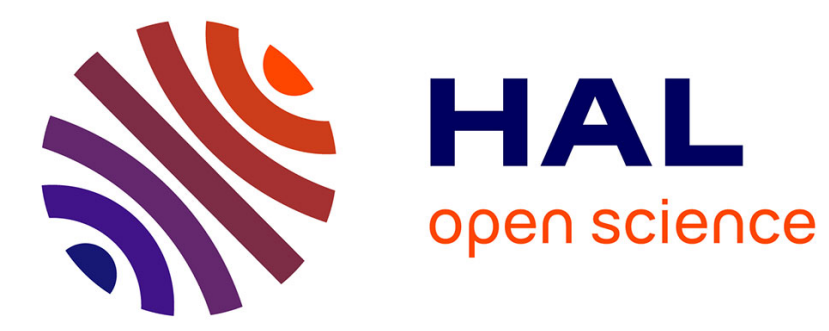

\title{
Coarse-Grained Molecular Dynamics for Copolymer-Vesicle Self-Assembly. Case Study: Sterically Stabilized Liposomes
}

\author{
Alexander Kantardjiev, Pavletta Shestakova
}

\section{- To cite this version:}

Alexander Kantardjiev, Pavletta Shestakova. Coarse-Grained Molecular Dynamics for CopolymerVesicle Self-Assembly. Case Study: Sterically Stabilized Liposomes. CS-DC'15 World e-conference, Sep 2015, Tempe, United States. hal-01291109

\section{HAL Id: hal-01291109 \\ https://hal.science/hal-01291109}

Submitted on 20 Mar 2016

HAL is a multi-disciplinary open access archive for the deposit and dissemination of scientific research documents, whether they are published or not. The documents may come from teaching and research institutions in France or abroad, or from public or private research centers.
L'archive ouverte pluridisciplinaire HAL, est destinée au dépôt et à la diffusion de documents scientifiques de niveau recherche, publiés ou non, émanant des établissements d'enseignement et de recherche français ou étrangers, des laboratoires publics ou privés. 


\title{
Coarse-Grained Molecular Dynamics for Copolymer- Vesicle Self-Assembly. Case Study: Sterically Stabilized Liposomes.
}

\author{
Alexander Kantardjiev ${ }^{1}$ and Pavletta Shestakova ${ }^{1}$ \\ ${ }^{1}$ Institute of Organic Chemistry with Centre of Phytochemistry, NMR Lab, \\ Bulgarian Academy of Sciences \\ alexkant@orgchm.bas.bg,psd@orgchm.bas.bg
}

\begin{abstract}
We carried out a series of Coarse-Grained Molecular Dynamics simulations of liposome-copolymer systems in an attempt to understand the effect of copolymer structure and concentration on vesicle closure. Coarse graining implies fewer degrees of freedom, cheaper potential calculation, smoother potential surfaces and larger time steps. In this way reliability and accuracy of fine grained (FG) i.e. atomistic models is combined with the efficiency of a reduced representation. Such a powerful approach helped our million atoms simulations to yield hundreds of nanoseconds trajectories starting from a randomized distribution of lipid molecules DPPC and Cholesterol plus varying concentration of copolymers with lipid mimetic units in explicit solvent (order of million water molecules). Simulation data is successfully verified against experimental DOSY-NMR results. The consistency of the theoretical evidence and experimental observation provides insight into the factors governing liposome-polymer stability with both fundamental and practical consequences.
\end{abstract}

\section{Introduction}

Our study aims to understand the principles governing polymer-lipid vesicle liposome assembly and ensuing hybrid liposome stability by combination of coarsegrained molecular dynamics simulations and complementary NMR-DOSY experiments. Besides fundamental interest (principles of vesicle formation and evolution) the problem has a strong application side - self-assembly of synthetic vesicles for nanotechnology [1]. A prominent example for the nano-technological interest in liposome systems is the intelligent design of drug delivery systems that require polymer stabilized vesicles. Such sterically stabilized liposomes fall within mesoscopic ordering of scales and thus the problem falls within spatial and temporal scales extending far beyond current computational capability for atomistic dynamics. 


\subsection{Some Musings About Simulation of Hybrid Vesicles}

A first guess approach is to apply coarse-graining which allows to tackle consistently the problem with adequate system size and time scale. Coarse graining implies fewer degrees of freedom, cheaper potential calculation, larger time step. In this way reliability and accuracy of fine grained (FG) i.e atomistic models is combined with the efficiency of a reduced representation. Thus our million-atoms simulations yield trajectories starting from a randomized distribution of lipid molecules (1,2Dipalmitoyl-sn-glycero-3-phosphocholine - DPPC and Cholesterol) and varying concentration of copolymers with lipid mimetic units in explicit solvent (order of million water molecules). Polyethylene glycol (PEG) grafted on vesicle surface has a stabilizing effect and leads to prolonged circulation times of the liposome in the blood stream. PEG covalently coupled to a phospholipid moiety, known as PEG-lipid, possesses the status of the current most effective steric stabilizer for liposomes. However conventional PEG-lipids suffer severe limitations due to the addition of a negative net charge stemming from the carbamate linkage (the covalent link of the PEG chain). On the other hand an ether bond (PEG moiety linked to the glycerol skeleton via ether inkages) leads to changes in the dipole potential. Such effects can be detrimental to some properties of of the sterically stabilized liposomes (e.g.their $\mathrm{pH}$-dependence). Here we focus on a new class promising stabilized - copolymers with ethylene oxide (EO). Short blocks of aliphatic double chains mimic lipid tails. The $\mathrm{C} 12$ aliphatic chains are attached to a glycerol skeleton via ether linkages. The PEG chain can be of variable length and this fact is to be exploited for search of a better stabilizing agent. Again, ether bonds are used to attach PEG chains to the lipidmimetic units.

\subsection{Thesis Proposal}

Our work is focused at computational and DOSY-NMR study of these novel copolymers with the following basic premise in mind - the number of the lipidmimetic anchors and the size of the PEG chain control the stabilizing potential of the copolymer. The number of lipid mimetic units is a prerequisite for pushing the saturation limit and thus enhancing the formation of a denser protective PEG layer around the vesicle. We used the following copolymers based on 1,3-didodecyloxypropane-2-ol (DDP) and 1,3-didodecyloxy-2-glycidylglycerol (DDGG) lipid mimetic anchors. Next we deal with methodological issues regarding MD membrane simulations - MD setup, coarse grained representations of lipids and observing properties computationally. 

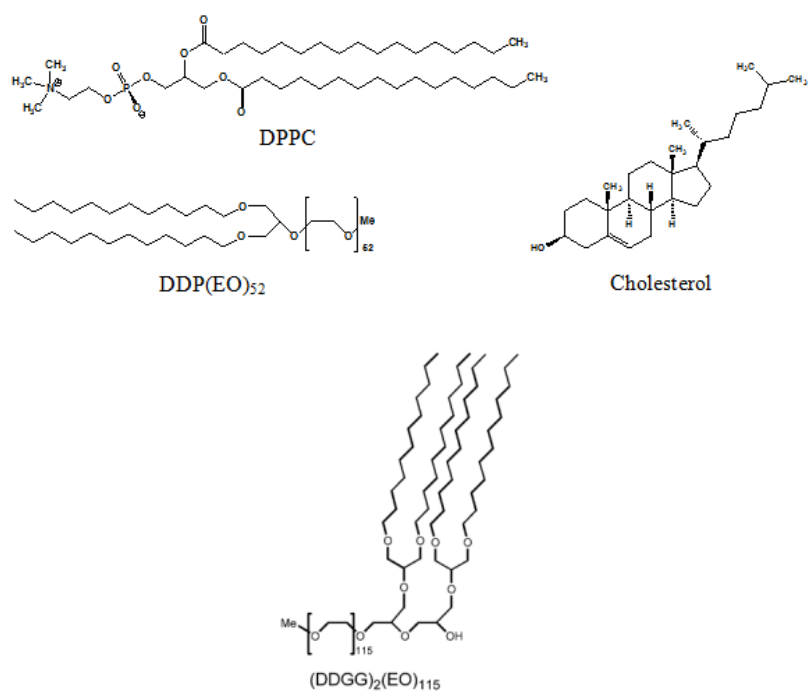

Fig. 1. Chemical structures of copolymer-vesicle constituents.

\section{Methods}

\subsection{Molecular Dynamics Setup}

Molecular dynamics simulations were carried out under the isothermal-isobaric conditions in order to emulate NPT ensemble. Pressure was fixed at 1.01 bars and coupled isotropically with a compressibility of $3 \times 10^{-3}$ bar $^{-1}$. We applied Berendsen barostat with a coupling constant of 5 ps. Velocity Verlet algorithm was used to propagate the equations of motion. A time step of $20 \mathrm{fs}$ was used. Actually a time stem of 40 fs was initially set (as recommended) but the stability of the simulation was compromised (due to heating that goes beyond the capability of the thermostat). Equilibration of the systems was monitored by looking at the system energy and some structural parameters as for example area per lipid. A cutoff of $12 \AA$ was used for the Lennard-Jones potential and electrostatic interactions.

\subsection{Coarse Grained Models of Major Player Molecules}

All systems were modeled with the MARTINI force field [2,3] which is reasonably close to the atomic details. Its scaling is nearly 4-fold (with some exceptions mentioned below). On average four heavy atoms are mapped to a single coarse-grain bead - interaction center. The philosophy is based on a modular principle by definition of an array of particle-bead types with specific chemical properties. As is the case with fine-grained atomistic case - the strength of the interaction is a function of the interacting particle types and the distance. Bonds and bond angles are described 
with a set of standard bonded potential energy functions-harmonic potentials for bonds / cosine type potential for angles. In our case MARTINI force field is used to model liposome assembly of DPPC / Cholesterol / Copolymer (DDP / DDGG) in explicit water molecules. DDP and DDGG polymers were modeled by combining the available parametrization for the PEG chain and the structure of the glycerol skeleton and corresponding aliphatic chains .

\subsection{Molecular Dynamics Simulation Software}

Molecular dynamics simulations were performed using the Gromacs software simulation package [4]. The software was installed in a GPU CUDA computer environment running Scientific Linux 6.3 as operating system. Each simulation was run using a 4 GPU nodes. Each node was equipped with Intel processors at $2.66 \mathrm{GHz}$ and $16 \mathrm{~GB}$ local memory (2GB per processor core).

\section{Results and Discussion}

\subsection{System Setup Organization}

A random ternary mixture of DPPC, Cholesterol, DDP / DDGG molecules in ratio corresponding to the experimental mol \% was generated by the genbox utility of Gromacs i.e. without a biased coarse-grained lipid template to construct the vesicle and therefore closer to the initial experimental setup. Phospholipids (DPPC) and cholesterol molecules were mixed in 2:1 molar ratio. We generated a concentration ladder of the selected promising copolymers - in MD search for the saturation limit and detailing the dependence of liposome assembly on concentration and structure of copolymers - DDP (EO) 52 or DDGG (EO) 115 or (DDGG) 2 (EO) 115 . A rectangular (cubic) box with a $20 \mathrm{~nm}$ size was used. Periodic boundary conditions were applied in all directions. The mix was solvated via the available Gromacs package utility (genbox). The initial configuration of the system was subjected to a round of energy minimization using the steepest descent algorithm until reaching a predefined cutoff (in effect - several hundred minimization steps). For such a system the corresponding atomistic representation would require on the order of million particles (atoms) and the prohibitive time step of 2 fs thus obliterating achieving adequate results with the available contemporary computational resources. Membranes with several species (components) with fixed proportions are tricky for the equilibration and in our setup it required hundreds of nanoseconds. We have calculated the average the order parameter through the averaging of the second Legendre polynomial over all lipids and time for each of the described vectors.

\subsection{Spontaneous Self-assemblies within hundreds of nanoseconds}

The process of self-assembly to vesicle closures and concurrent bicelles / micelle structures is followed along several hundreds of nanoseconds long MD trajectories. The concise format of this contribution prohibits presentation of the overwhelming 
amount of data - thus we reveal several informative snapshots which directly support the hypothesis of the "PEG Length to Lipid Mimetics" ratio as a major structural determinant controlling the shielding property of the copolymer in vesicle assembly.

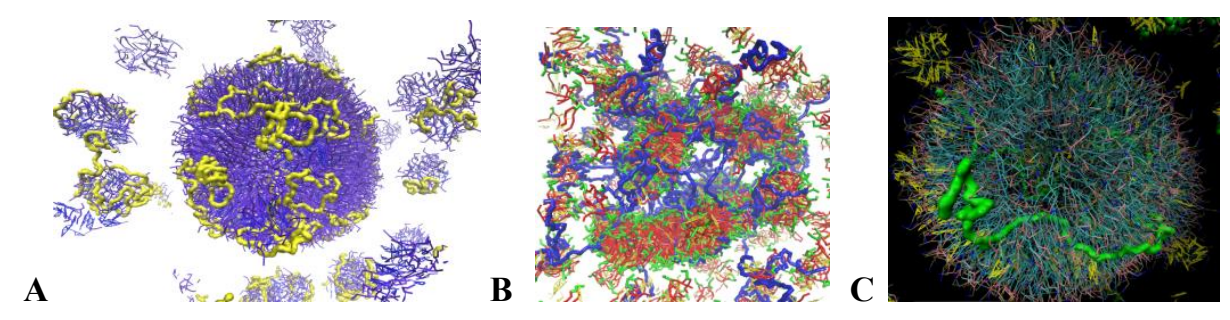

Fig. 2. Unequivocal direct support for the "PEG length vs Lipid Mimetics" ratio hypothesis. Major players at around $100 \mathrm{~ns}$ for a $5 \mathrm{~mol} \%$ copolymer concentration with respect to DPPC.

A. DDP (EO) 52 "PEG-52 vs single DDP lipid-mimetics". DDP (EO)s2 - yellow.

B. DDGG (EO) ${ }_{115}$ "PEG-115 vs single DDGG lipid-mimetics". DDGG (EO) 115 is in blue; DPPC heads are green; DPPC tails are red. Bicelle refused closure to a vesicle.

C. $(\mathrm{DDGG})_{2}(\mathrm{EO})_{115}$ "PEG-115 vs double DDGG lipid-mimetics". (DDGG)2 $(\mathrm{EO})_{115}$ is in green. Restored potency for vesicle formation due to the additional lipid mimetic.

\subsection{Credulity Addendum to MD Simulations: Coincidence with DOSY-NMR Data}

For a specific DDP (EO) 52 concentration (a 5 mol\%) a DOSY NMR experiment was carried out. Simulation data is successfully verified against the experimental NMR results. 


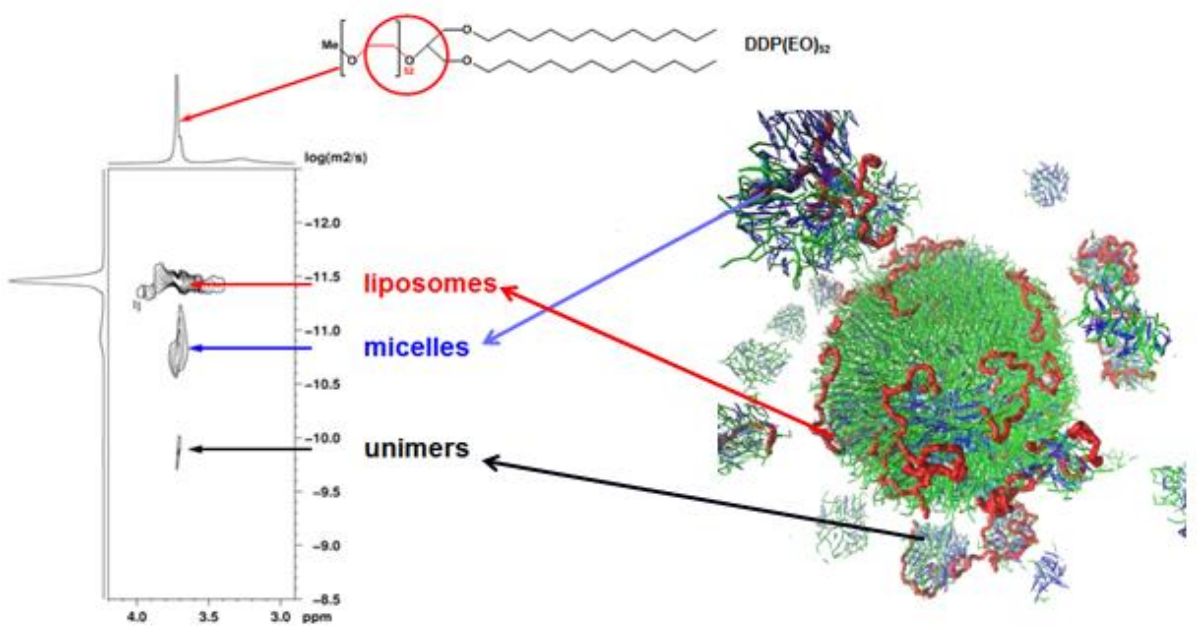

Fig. 3. DOSY-NMR spectra of a DDP(EO) $)_{52}$ Copolymer-Liposome against a simulation snapshot at around 100 ns with the same components and concentrations. Nice fit of both the liposome itself and the concurrent satellite structures.

\section{Conclusions}

1. "PEG length vs Lipid Mimetics" ratio might be a determinant for copolymer "stealth" potential.

2. Consistency with NMR-DOSY findings

\section{References}

1. Lasic DD, Needham D. Stealth liposomes: a prototypical biomaterial. Chem Rev 1995,95:2601-2628

2. Marrink, S. J., De Vries, A. H., and Mark, A. E. (2004) Coarse grained model for semiquantitative lipid simulations. J. Phys. Chem. B 108, 750-760

3. Marrink, S. J., Risselada, H. J., Yefimov, S., Tieleman, D. P., and De Vries, A. H. (2007) The MARTINI force field: coarse grained model for biomolecular simulations. J. Phys. Chem. B 111, 7812-7824.

4. D. van der Spoel, E. Lindahl, B. Hess, G. Groenhof, A. E. Mark and H. J. C. Berendsen, J. Comput. Chem., 2005, 26, 1701

5. Humphrey, W., Dalke, A. and Schulten, K., "VMD - Visual Molecular Dynamics", J. Molec. Graphics, 1996, vol. 14, pp. 33-38. 